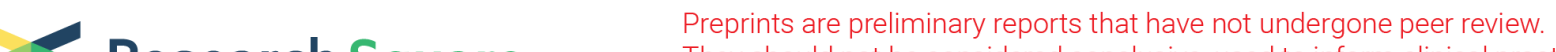 Research Square They should not be considered conclusive, used to inform clinical practice, or referenced by the media as validated information.
}

\section{Neurofilament degradation is involved in laparotomy-induced cognitive dysfunction in aged rats}

\section{Yiyun Cao}

Shanghai 6th Peoples Hospital Affiliated to Shanghai Jiaotong University School of Medicine

Taotao Liu

Peking University Third Hospital

\section{Zhengqian Li}

Peking University Third Hospital

\section{Jiao Yang}

Shanghai 6th Peoples Hospital Affiliated to Shanghai Jiaotong University School of Medicine

\section{Lijun Ma}

North Minzu University

\section{Aihua Qi}

Shanghai 6th Peoples Hospital Affiliated to Shanghai Jiaotong University School of Medicine

\section{Feng Zou}

Shanghai 6th Peoples Hospital Affiliated to Shanghai Jiaotong University School of Medicine

\section{Xiangyang Guo}

Peking University Third Hospital

\section{Aizhong Wang ( $\square$ pencil6699@163.com )}

Shanghai 6th Peoples Hospital Affiliated to Shanghai Jiaotong University School of Medicine https://orcid.org/0000-0002-2210-2505

\section{Research}

Keywords: Postoperative cognitive dysfunction (POCD), neuroinflammatory, laparotomy, neurofilaments (NFs)

Posted Date: February 26th, 2020

DOI: https://doi.org/10.21203/rs.2.24620/v1

License: (c) (i) This work is licensed under a Creative Commons Attribution 4.0 International License. Read Full License 
Version of Record: A version of this preprint was published at Aging on November 24th, 2020. See the published version at https://doi.org/10.18632/aging.104172. 


\section{Abstract}

Background An excessive neuroinflammatory response involved in the pathogenesis of postoperative cognitive dysfunction (POCD), which increases morbidity and mortality. However, the precise mechanism remains unclear. Trichostatin A (TSA), a histone deacetylase inhibitor, has been shown to be antiinflammatory. Therefore, we aimed to explore whether TSA can inhibit the surgery-induced neuroinflammation and improve POCD and further reveal the complex neuropathogenesis underlying POCD.

Methods To explore the molecular mechanisms by which surgery-induced POCD in aged rats, TSA (1 $\mathrm{mg} / \mathrm{kg}$ ) was intraperitoneally injected, and hippocampal microglial activation and neuroinflammation were observed. We investigated changes in the protein profile of the hippocampus using a proteomics approach [isobaric tags for relative and absolute quantitation (iTRAQ) combined with nano liquid chromatography-mass spectrometry] at the peak of surgery-induced neuroinflammation, and significant alterations of proteins were verified using western blotting and immunofluorescence. Then, proteins associated with signaling pathways in the surgery + TSA and surgery groups were analyzed using the Kyoto Encyclopedia of Genes and Genomes (KEGG).

Results After laparotomy, aged rats had prolonged escape latencies on days 4 and 5 postsurgery, spent less time in the target quadrant than control rats $(p<0.05)$, and exhibited excessive hippocampal microglia activation and IL-1 $\beta$ and TNF- $a$ release. ITRAQ and bioinformatics analyses at $6 \mathrm{~h}$ after surgery showed that neurofilaments (NFs), including the NEFH, NEFM and NEFL proteins, were significantly upregulated, and TSA pretreatment could mitigate these changes. Subsequently, KEGG analysis revealed that nine pathways were enriched in the surgery + TSA group vs. the surgery group $(p<0.05)$. Among them, two signaling pathways, "focal adhesion" and "ECM-receptor interaction", were associated with significant upregulation of collagen and downregulation of NF proteins, indicating these as possibly important pathways involved in NF degradation in the hippocampus of aged brains after surgery-induced POCD.

Conclusion Surgery-induced neuroinflammation upregulated NFs, resulting NF degradation and aggregation in the hippocampus of aged rats, which might lead to hippocampus-independent learning and memory impairment, contributing to POCD. Additionally, TSA diminished surgery-induced neuroinflammatory responses and modulated the NF-associated changes in cognitive dysfunction in aged brains, which might be related to activation of the "focal adhesion" and "ECM-receptor interaction" pathways.

\section{Introduction}

Postoperative cognitive dysfunction (POCD) commonly occurs in older adults after surgery and is frequently under-recognized. Development of POCD has been associated with worse outcomes, longer hospital stays, decreased quality of life, increased mortality and risk of dementia. POCD has a high 
incidence $(10 \%-54 \%)$ and is currently more often recognized clinically because of an increased awareness [1, 2]. In recent years, many studies have suggested that the pathogenesis of POCD is a synergistic effect of various factors, including tau phosphorylation, amyloid $\beta$ accumulation [3], blood brain barrier (BBB) disruption [4] and immune system disorders $[5,6]$. However, the precise pathophysiology of POCD remains unclear.

The hippocampus is an important component of the limbic system and is associated with the regulation of learning, memory and behaviors. Our previous studies revealed that laparotomy could increase the proinflammatory cytokines interleukin-1 $\beta$ (IL-1 $\beta$ ) and tumor necrosis factor-alpha (TNF- $\alpha$ ) in the hippocampus and cause cognitive dysfunction to persist for several weeks in aged rats [7]. However, the precise mechanism of this neuroinflammatory reaction, which has been confirmed to be a key factor mediating the development of POCD, and its extensive cross-reaction with other elements in hippocampal neurons remain elusive and need to be further explored.

Dysfunction of microglia is involved in the amplification of the immune response in the pathophysiology of neurological disorders, including ischemic stroke [8] and neurodegenerative diseases $[9,10]$. Overexpression of inflammatory cytokines in the brain after surgery and anesthesia exposure $[5,6]$ can be caused by microglial release of cytokines [11]. Microglia constitute $10 \%-15 \%$ of the glial cell population in the parenchyma in the adult brain [12]. Hence, anti-inflammatory therapies can alleviate the progression of brain disorders. Trichostatin A (TSA), a histone deacetylase (HDAC) inhibitor, is also associated with an anti-inflammatory effect [13], but its role in surgery-induced neuroinflammation in aged brains is unclear. Hence, our study explored whether TSA can reduce microglia-mediated neuroinflammation and improve surgery-induced cognitive dysfunction as well as the possible mechanism.

In the present study, a surgery model was established in aged rats by isoflurane exposure. This study aimed to examine the dynamic changes in microglial activation, surgery-induced hippocampal neuroinflammation and cognitive impairment in aged rats. Then, the isobaric tags for the relative and absolute quantitation (iTRAQ) method combined with nano liquid chromatography-mass spectrometry (NanoLC-MS/MS), developed for protein quantitation, was used to reveal the profile of differentially altered proteins in the hippocampus underlying the animal model of POCD, and bioinformatics analyses were performed to explore the correlated signaling pathway. The study aimed to reveal the pathogenesis of POCD and provide potential therapeutic targets for its prevention and treatment in the vulnerable older brain.

\section{Materials And Methods}

\subsection{Animals}

Aged male Sprague-Dawley rats (20 months old; weight, 550-650 g) were used for all experiments. Animals were purchased from the Dongchuang Laboratory Animal Center (Changsha, Hunan, China) and bred under standardized housing conditions with ad libitum access to food and water. All rats were 
allowed to adapt to their new environment for at least 7 days before beginning experiments. The experimental protocol was approved by the Institutional Animal Care and Use Committee of the Sixth People's Hospital Affiliated with Shanghai Jiao Tong University (SYXK [Shanghai, China] 2016-0020, 22 February 2017).

\subsection{Experimental protocol}

\subsubsection{Effect of TSA block on spatial learning and memory}

The effects of TSA (Sigma, St, Louis, MO) on surgery-induced (laparotomy) cognitive decline were examined. Rats were randomly assigned to control, surgery, surgery + TSA and TSA groups ( $n=12$ each). Rats in the surgery + TSA and TSA groups were intraperitoneally administered $1 \mathrm{mg} / \mathrm{kg}$ TSA $30 \mathrm{~min}$ before surgery; this dosing protocol has been shown to effectively protect against kainic acid-induced memory deficits [14]. TSA was dissolved in a vehicle solution (1 mg/ml in 10\% DMSO). Rats in the other two groups received an identical volume of vehicle solution. Following the pretreatment phase, the animals either underwent laparotomy or sham surgery under isoflurane anesthesia. Spatial learning and memory were then tested using the Morris water maze (MWM) task.

\subsubsection{Effects of laparotomy on physiological parameters in aged rats}

To determine whether isoflurane anesthesia and laparotomy caused physiological side effects such as hypoxia, hypercapnia or hypoglycemia, five rats in the various treatment groups were selected as cardiorespiratory control animals (total: $n=20)$. After the surgery, blood samples $(0.5 \mathrm{ml})$ were immediately collected for arterial blood gas (OPTI Medical Systems, Roswell, GA) and blood glucose (Life Scan Inc., Milpitas, CA) analysis. The cardiorespiratory control rats were not used for any other part of the study.

\subsubsection{Neuroinflammation and iTRAQ-based proteomics in the hippocampus after surgery}

To study the effects of peripheral surgical trauma on microglia activity in the brain, rats were randomly assigned to control, surgery, surgery + TSA and TSA groups and received laparotomy or sham operations. Markers for microglial activation in the hippocampus were dynamically determined at 6,24 and $72 \mathrm{~h}$ after surgery using western blotting and immunofluorescence ( $n=5$ per time point).

In the present study, we found that expression of Iba-1 (a microglial cell activation marker) was significantly increased at $6 \mathrm{~h}$ after laparotomy compared with other observational time points, indicating that this marker peaked at $6 \mathrm{~h}$ after surgery; therefore, the effects of surgery on the protein profile alterations in the hippocampus were assessed at $6 \mathrm{~h}$ after surgery using iTRAQ ( $\mathrm{n}=4$ each). 


\subsection{Anesthesia and surgery}

Animals were exposed to $1.5 \%$ isoflurane for $5 \mathrm{~min}$ in a small chamber and then removed and endotracheally intubated [15]. The laparotomy was aseptically performed under mechanical ventilation using a previously described method $(1 \%-2 \%$ isoflurane in $100 \%$ oxygen) that was developed to model POCD in aged rats [16]. Briefly, with the surgeon wearing sterile latex gloves, the abdominal region was shaved and sterilized. A 3-cm vertical incision was made approximately $0.5 \mathrm{~cm}$ below the lower right rib. The viscera and incised muscle were vigorously manipulated by inserting an index finger up to the second knuckle into the opening for $30 \mathrm{~s}$. Next, approximately $10 \mathrm{~cm}$ of the intestine was exteriorized and vigorously rubbed with the thumb and index finger, also for $30 \mathrm{~s}$, and then placed back into the cavity. Finally, the surgeon separately sutured the peritoneal lining, abdominal muscle and skin. The laparotomy duration was 20-25 min. The sham operation group was treated in an identical manner for the same amount of time, except that laparotomy was not performed.

\subsection{Enzyme-linked immunosorbent assays (ELISAs)}

Expression of proinflammatory cytokines in the hippocampus was determined with an ELISA (IBL, Takasaki, Japan). The hippocampus was separated, homogenized in extraction buffer and centrifuged, and the total protein concentration of the supernatant was determined using a bicinchoninic acid (BCA) protein assay kit. Then, $100 \mu \mathrm{l}$ of the supernatant was collected and analyzed by an ELISA according to the manufacturer's instructions. The results were assayed at $450 \mathrm{~nm}$, and data are expressed as pg/mg of tissues.

\subsection{Western blot}

Western blots were performed as previously described [4]. The primary antibodies used included anti-lba1 (1:1000; Abcam, San Diego, CA); anti-neurofilament (NF) light chain (anti-NEFL) (1:1000; Abcam); antiNF medium chain (anti-NEFM) (1:1000; Abcam); and anti-NF heavy chain (anti-NEFH) (1:1000; Abcam). Fluorescently labeled secondary antibodies (1:10,000; LI-COR Biosciences, Lincoln, NE) were also used.

\subsection{Immunofluorescence}

Immunofluorescence staining was performed as previously described [4]. Briefly, after incubation in the primary antibody, including anti-lba-1 (1:1,000; Abcam), anti-NEFL (1:500; Abcam), anti-NEFM (1:500; Abcam), and anti-NEFH (1:100; Abcam), the signal was detected with a fluorescein isothiocyanate-labeled secondary antibody (1:200; Abcam). The nuclei were counterstained with 4, 6-diamidino-2-phenylindole 
(DAPI) (1:5,000; Roche, Mannheim, Germany). Images were acquired on a Leica DM3000 fluorescence microscope (Leica, Wetzlar, Germany).

\section{7. iTRAQ labeling and NanoLC-MS/MS analysis}

Hippocampus tissues were ultrasonically disrupted in lysis buffer (Roche) on ice. Supernatants were collected after centrifugation $\left(10,000 \mathrm{~g}, 30 \mathrm{~min}, 4^{\circ} \mathrm{C}\right)$, and protein concentrations were determined using an enhanced BCA Protein Assay Kit (P0010; Beyotime Biotechnology Ltd., Beijing, China) following the manufacturer's instructions. The protein samples $(200 \mu \mathrm{g})$ were mixed with dl-dithiothreitol, alkylated with iodoacetamide and then digested in trypsin (protein-trypsin ratio $=50: 1,12 \mathrm{~h}$ ). Then, the peptides were labelled with an iTRAQ reagent-8-plex multiplex kit according to the manufacturer's instructions. Samples were labeled with the iTRAQ tags as follows: the control group (tags 113 and 117), the surgery group (tags 115 and 119), the surgery + TSA group (tags 116 and 121) and the TSA group (tags 114 and118). All labelled samples were mixed and dried by vacuum centrifugation (EYELA, Tokyo, Japan).

The peptides were re-dissolved in $30 \mu \mathrm{l}$ of solvent A (A: $0.1 \%$ formic acid in water) and analyzed by on-line nanospray LC-MS/MS on an Orbitrap Fusion ${ }^{\text {TM }}$ instrument coupled to an EASY-nLC 1200 system (Thermo Fisher Scientific, MA, USA). The peptide sample $(4 \mu \mathrm{l})$ was loaded (trap column (Thermo Fisher Scientific Acclaim PepMap C18, $100 \mu \mathrm{m} \times 2 \mathrm{~cm}$ ), analytical column (Acclaim PepMap C18, $75 \mu \mathrm{m} \times 15 \mathrm{~cm}$ )) and separated with a linear gradient, ranging from $3 \%$ B (B: $0.1 \%$ formic acid in $A C N)$ to $32 \%$ B in 120 min. The column flow rate was maintained at $300 \mathrm{nl} / \mathrm{min}$ with a column temperature of $40^{\circ} \mathrm{C}$. An electrospray voltage of $2 \mathrm{kV}$ vs. the inlet of the mass spectrometer was used.

The mass spectrometer was run in the data-dependent acquisition mode and automatically switched between the MS and MS/MS mode. The parameters were as follows: (1) MS: scan range $(\mathrm{m} / \mathrm{z})=350-$ 1550; resolution $=60,000 ; \mathrm{AGC}$ target $=4 \mathrm{e} 5$; maximum injection time $=50 \mathrm{~ms}$; included charge states $=$ 2-6; dynamic exclusion = $45 \mathrm{~s}$; (2) HCD-MS/MS: resolution = 30,000; isolation window = 1.2; AGC target = 7e4; maximum injection time $=100 \mathrm{~ms}$; collision energy $=38$.

\subsection{MS data analysis}

Tandem mass spectra were processed by PEAKS Studio version 8.5 (Bioinformatics Solutions Inc., Waterloo, Canada). PEAKS DB was set to search the UniProt-Rat database (30226 entries, ver 201708) using trypsin as the digestion enzyme. The PEAKS DB search was performed with a fragment ion mass tolerance of $0.05 \mathrm{Da}$ and a parent ion tolerance of $7 \mathrm{ppm}$. Carbamidomethylation (C) and iTRAQ 8plex (K, $\mathrm{N}$-term) were specified as the fixed modifications. Oxidation (M), Deamidation (NQ), and Acetylation (Protein N-term), were specified as the variable modifications. Peptides were filtered with a $1 \%$ FDR, and a unique peptide was specified. PEAKSQ was used to calculate peptide and protein abundance. Normalization was performed when averaging the abundance of all peptides. Medians were used for 
averaging. Differentially expressed proteins were filtered if their fold change was greater than 1.2 and they contained at least two unique peptides with a significance value greater than $13(p<0.05)$.

\subsection{Bioinformatics analysis}

Blast2GO version 4 was used for functional annotation. The whole protein sequence database was analyzed by BlastP, and the results were mapped and annotated with the Gene Ontology database. Functional statistics of differentially expressed proteins were calculated by Fisher's exact test in Blast2GO. Pathway analysis was performed using the Kyoto Encyclopedia of Gene and Genomes (KEGG) and was processed by KOBAS (http://kobas.cbi.pku.edu.cn/). Functional protein association networks were generated using STRING.

\subsection{MWM test}

The MWM test was performed 2 days after surgery (allowing for abdominal incision healing) and conducted by investigators blinded to the group conditions as previously described [7]. Swimming was tracked by video (Sunny Instruments Co. Ltd., Beijing, China). The latency, swim speed, time to first platform crossing and time spent in the previous platform quadrant were analyzed.

\subsection{Statistical Analysis}

Statistics were calculated using SPSS 16.0 for Windows (SPSS, Inc., Chicago, IL, USA). Data on escape latency in the MWM tests were analyzed with two-way repeated-measures ANOVA followed by a post-hoc Bonferroni test. All other quantitative data were analyzed with by one-way ANOVA using Bonferroni posthoc analysis. Statistical significance was set at $p<0.05$. All data are shown as means \pm SEM (standard error of the mean).

\section{Results}

\subsection{TSA pretreatment improves cognitive function after surgery}

During the MWM test, we found that aged rats in the surgery group showed longer escape latencies than those in the control group, the surgery + TSA group and TSA group (Fig. $1 \mathrm{~A} ; \mathrm{p}<0.05$ ); No significant difference was observed in latencies among the control, the surgery + TSA group and TSA groups. All aged rats appeared to swim normally, and swimming speeds were not different among groups (Fig. $1 \mathrm{~B} ; \mathrm{p}$ $>0.05$ ). In the probe test, rats in the surgery group required a longer time to achieve their first platform 
crossing (Fig. $1 \mathrm{C} ; \mathrm{p}<0.05$ ), and the time spent in the target quadrant was much shorter than that of rats in the control group (Fig. $1 \mathrm{D}-\mathrm{E} ; \mathrm{p}<0.05$ ), confirming the presence of memory impairments after surgery. All of these changes were significantly alleviated by TSA pretreatment, suggesting that TSA improves cognition.

\subsection{TSA inhibits surgery-induced microglia activation}

Microglia mediate cytokine expression in the CNS, and limiting microglial activity is considered beneficial to reduce neuroinflammation [17]. The effects of TSA on surgery-induced microglial activation were determined. The results of the immunofluorescent examination showed that Iba- 1 levels were significantly higher in the hippocampal CA1 region (arrowheads) of surgery-only challenged rats and were reduced in rats pretreated with TSA at 6,24 and $72 \mathrm{~h}$ postsurgery (Fig. $2 \mathrm{~A}$ ). The western blotting results confirmed that hippocampal Iba-1 protein expression peaked at $6 \mathrm{~h}$ and decreased within $72 \mathrm{~h}$ postsurgery compared with the controls (Fig. 2 B and C; $p<0.001$ and $p<0.05$ ). Again, lba-1 protein expression was significantly higher in surgery-only-treated than in the control, surgery + TSA-treated and TSA-treated rats at 6, 24 and $72 \mathrm{~h}$ postsurgery (Fig. $2 \mathrm{D}-\mathrm{l} ; \mathrm{p}<0.05$ ). Hippocampal lba-1 protein levels were not altered following TSA-only treatment. These results indicated that surgery induced microglia activation and that this was prevented by TSA in aged rats.

\subsection{TSA inhibits surgery-induced neuroinflammation}

TSA modulates cytokine synthesis and release [13]. We thus determined the effects of TSA on surgeryinduced proinflammatory cytokine expression. Beginning at $6 \mathrm{~h}$ after surgery, the expression levels of IL$1 \beta$ and TNF- $\alpha$ in the hippocampus were significantly increased until $72 \mathrm{~h}$ after surgery (Fig. $3 \mathrm{~A}$ and $\mathrm{B}, \mathrm{p}$ $<0.05)$. In contrast, TSA treatment resulted in a significant reduction in IL-1 $\beta$ and TNF- $\alpha$ expression levels within $72 \mathrm{~h}$.

\subsection{TSA inhibits surgery-induced neurofilaments (NFs) upregulation}

Compared with the control, surgery + TSA and TSA groups, the results from the iTRAQ analyses of proteins in the surgery group, including the intermediate filament (IF) rod domain profile (ProSiteProfiles), IF protein (Pfam), and IF rod domain signature showed fold changes were greater than 1.4, and these proteins exhibited significant increases in IPR039008 (ProSiteProfiles), IPR039008 (Pfam) and PR018039 (ProSitePatterns) peptides $(p=0.01570)$ at $6 \mathrm{~h}$ after surgery (Fig. 4 A).

Based on the results of the MS analysis, the expression levels of three dysregulated proteins (NEFH, NEFM and NEFL) at $6 \mathrm{~h}$ after surgery were validated using immunofluorescence (Fig. 4 B) and western 
blotting (Fig. $4 \mathrm{C}$ and D). These three proteins were significantly upregulated in the surgery-treated group compared with the control group, and TSA pretreatment significantly prevented the surgery-induced increase in these proteins in the hippocampus. When given alone, TSA had no effect on the expression of $\mathrm{NEFH}, \mathrm{NEFM}$ and NEFL at $6 \mathrm{~h}$ after surgery. Therefore, the altered expression levels of proteins were consistent with the results from the MS analysis.

\subsection{KEGG pathways analysis}

An analysis of significantly differentially expressed proteins in the two groups was performed using the KEGG Pathway database (https://www.genome.jp/kegg/pathway.html). A total of 32 pathways were enriched in the surgery + TSA vs. the surgery group, and among them, nine pathways showed statistically significant enrichment (Fig. 5 A and B, p < 0.05), including focal adhesion (rno04510), ECM-receptor interaction (rno04512), protein digestion and absorption (rno04974), AGE-RAGE signaling pathway in diabetic complications (rno04933), amoebiasis (rno05146), platelet activation (rno04611), glutamatergic synapse (rno04724), primary bile acid biosynthesis (rno00120), and phospholipase D signaling pathway (rno04072). Of these, the "focal adhesion" ( $p=0.005433)$ and "ECM-receptor interaction" ( $p=0.007986)$ pathways were the top two significantly enriched pathways and were chosen for analysis. In Fig. $5 \mathrm{C}$, red represents upregulated proteins, and blue represents downregulated proteins.

\section{Discussion}

The laparotomy model was used in our study, which is a classic animal model that is widely used by researchers for POCD studies $[7,16]$. Accumulated evidence demonstrates that the interaction of microglia and inflammatory cytokines participates in the pathology of cognitive impairment [18]. However, the role that microglia play in surgery-induced neuroinflammation and how the precise progression contributes to cognitive dysfunction, as well as the related signaling pathways, remain unexplored. We have demonstrated for the first time that TSA pretreatment alleviates surgery-induced NFs damage in the hippocampus of aged rats by decreasing microglial activation and inflammatory factor (IL-1 $\beta$ and TNF- $\alpha$ ) release, as well as changing the cargo of the proteins associated with the extracellular matrix (ECM). These results support the notion that microglial activation and its derived neuroinflammation play a role in surgery-induced cognitive dysfunction. The results also provide some insight into the NFs damage triggered by neuroinflammation observed in the aged brain, which was suppressed by TSA through the "focal adhesion" and "ECM-receptor interaction" pathways, which are major pathways involved in this process.

Our data allow examination of the role of infiltrating immune cells in the pathogenesis of POCD. In neurodegenerative or neuroinflammatory diseases, the CNS exhibits increased activation of microglia and higher levels of proinflammatory cytokines such as TNF- $\alpha$ and IL-1 $\beta[19,20]$, which is consistent with our 
results. These cytokines promote the release of secondary inflammatory mediators including prostaglandins and nitric oxide [21], which are essential for the induction and maintenance of the behavioral symptoms of this condition [22]. According to our results, the aged rats exhibited deficits in hippocampus-dependent learning and memory after surgery. TSA, which can cross the BBB [14], can alleviate expression of Iba-1, a marker of microglia activation, and reduce the levels of the proinflammatory cytokines IL-1 $\beta$ and TNF- $\alpha$ in aged rat brains, improving surgery-induced cognitive dysfunction.

In order to further explore the differentially expressed proteins in the POCD model, iTRAQ analysis was performed on the hippocampus of aged rats at $6 \mathrm{~h}$ after laparotomy, which is the peak of surgery-induced neuroinflammation, and the NFs level in the surgery group was significantly higher than those in the other groups (Fig. 4). NFs, which are a type of IF, are polymers made from NFEL, NFEM, NFEH and a-internexin or peripherin; each subunit has various structural domains and functions [23]. Basal neurotransmission and induction of hippocampal long-term potentiation are abnormal in NFEM knockout mice, and NFEH knockout mice showed a marked decrease in conduction velocity in large myelinated axons [24]. Moreover, NFEL was significantly increased in the plasma of Alzheimer's disease patients $(149 \%$ vs. control) [25]. Based on the findings of previous studies, our results show that exposure of aged rats to surgery can induce NEFH, NEFM and NEFL upregulation (because of the degradation of NFs), resulting in NFs aggregation [23], thus reducing the NFs content. Therefore, the calibers of large axons can exhibit general axonal dysfunction and decreased conduction velocities, leading to hippocampal dysfunction, which is characterized by spatial learning and memory declines. In our present study, microglia were in the activated state in the aged rat hippocampus after laparotomy, which might be caused by IL-1 $\beta$ and contribute to NFs disorder [26]. The NFs expression level in the surgery group was much higher than those in the TSA pretreatment and control groups, indicating that TSA could attenuate the NFs degradation and aggregation in the hippocampus, which could improve surgically-induced cognitive dysfunction in the aged brain.

Nine different KEGG pathways were altered in the TSA + surgery group compared with the surgery group. In addition, the reactome pathway analysis showed a predominance of proteins involved in the "focal adhesion" and "ECM-receptor interaction" pathways, which are related to NFs degradation and the aggregation phase in the hippocampus after laparotomy, as evidenced by marked collagen upregulation and filamin downregulation (Fig. $5 \mathrm{~A}$ and B). Therefore, the present results showed that TSA clearly increased collagen protein expression in the hippocampus. Collagen is the main component of the ECM and provides the structural support that is required for normal ECM assembly, and it suppresses NF overexpression in response to surgery (Fig. $5 \mathrm{C}$ and D) via activation of the "focal adhesion" and "ECMreceptor interaction" signaling pathways, thus improving surgery-induced spatial memory impairment. We hypothesize that the accumulation of collagen might provide structural support to myelinated axons in the hippocampus and alleviate the degradation of NFs, protecting the normal conduction function of axons and increasing neuronal efficiency in the damaged circuit. However, this is only informed speculation because collagen has been previously reported to be one of the main materials that promotes repair of damaged nerves [27] and guides newborn NFs extension [28], thus enhancing 
nerve regeneration and functional recovery. Nevertheless, the role of significantly differentially expressed signaling pathway proteins in the improvement of cognitive dysfunction is not necessarily proportional to these differences, and further verification of which signaling pathways are more effective in improving surgery-induced POCD is still needed.

\section{Abbreviations}

POCD

postoperative cognitive dysfunction; TSA:Trichostatin A; NFs:neurofilaments; NEFL:neurofilament light chain; NEFM:neurofilament medium chain; NEFH:neurofilament heavy chain; BBB:blood brain barrier; IL1及:interleukin-1 $\beta$; TNF-a:tumor necrosis factor-alpha; HDAC:histone deacetylase; ECM:extracellular matrix; MWM:Morris water maze; iTRAQ:isobaric tags for relative and absolute quantitation; KEGG:Kyoto Encyclopedia of Genes and Genomes; NanoLC-MS/MS:nano liquid chromatography-mass spectrometry.

\section{Declarations}

\section{Acknowledgements}

Not applicable.

\section{Funding}

This work was supported by grants from the Shanghai Pudong New District Science and Technology Development Fund Medical and Health Project of China (No. PKJ2018-Y56).

\section{Availability of data and materials}

All data generated or analyzed during this study are included in this published article.

\section{Authors' contributions}

YYC, XYG and AZW conceived and designed the experiments; YYC, TTL, FZ, AHQ and JY performed the experiments; YYC and LJM analyzed the data; YYC and ZQL wrote the paper. All authors read and approved the final manuscript. 


\section{Ethics approval and consent to participate}

Our research was performed with the approval of the Sixth People's Hospital Affiliated with Shanghai Jiao Tong University Biomedical Ethics Committee Experimental Animal Ethics Branch (SYXK [Shanghai, China] 2016-0020, 22 February 2017).

\section{Consent for publication}

All named authors have given consent for publication.

\section{Competing interests}

The authors declare that they have no competing interests.

\section{References}

1. Inouye SK: Delirium in older persons. N Engl J Med 2006, 354:1157-1165.

2. Androsova G, Krause R, Winterer G, Schneider R: Biomarkers of postoperative delirium and cognitive dysfunction. Front Aging Neurosci 2015, 7:112.

3. Zhang B, Tian M, Zheng H, Zhen Y, Yue Y, Li T, Li S, Marcantonio ER, Xie Z: Effects of anesthetic isoflurane and desflurane on human cerebrospinal fluid Abeta and tau level. Anesthesiology 2013, 119:52-60.

4. Cao Y, Li Z, Li H, Ni C, Li L, Yang N, Shi C, Zhong Y, Cui D, Guo X: Hypoxia-inducible factor-1alpha is involved in isoflurane-induced blood-brain barrier disruption in aged rats model of POCD. Behav Brain Res 2017.

5. Nemeth E, Vig K, Racz K, Koritsanszky KB, Ronkay KI, Hamvas FP, Borbely C, Eory A, Merkely B, Gal J: Influence of the postoperative inflammatory response on cognitive decline in elderly patients undergoing on-pump cardiac surgery: a controlled, prospective observational study. BMC Anesthesiol 2017, 17:113.

6. Li ZQ, Rong XY, Liu YJ, Ni C, Tian XS, Mo N, Chui DH, Guo XY: Activation of the canonical nuclear factor-kappaB pathway is involved in isoflurane-induced hippocampal interleukin-1beta elevation and the resultant cognitive deficits in aged rats. Biochem Biophys Res Commun 2013, 438:628-634.

7. Li Z, Cao Y, Li L, Liang Y, Tian X, Mo N, Liu Y, Li M, Chui D, Guo X: Prophylactic angiotensin type 1 receptor antagonism confers neuroprotection in an aged rat model of postoperative cognitive dysfunction. Biochem Biophys Res Commun 2014, 449:74-80.

8. Zhao SC, Ma LS, Chu ZH, Xu H, Wu WQ, Liu F: Regulation of microglial activation in stroke. Acta Pharmacol Sin 2017, 38:445-458. 
9. Sims R, van der Lee SJ, Naj AC, Bellenguez C, Badarinarayan N, Jakobsdottir J, Kunkle BW, Boland A, Raybould R, Bis JC, et al: Rare coding variants in PLCG2, ABI3, and TREM2 implicate microglialmediated innate immunity in Alzheimer's disease. Nat Genet 2017, 49:1373-1384.

10. Heneka MT, Kummer MP, Latz E: Innate immune activation in neurodegenerative disease. Nat Rev Immunol 2014, 14:463-477.

11. Cherry JD, Olschowka JA, O'Banion MK: Neuroinflammation and M2 microglia: the good, the bad, and the inflamed. J Neuroinflammation 2014, 11:98.

12. Nayak D, Roth TL, McGavern DB: Microglia development and function. Annu Rev Immunol 2014, 32:367-402.

13. Kannan V, Brouwer N, Hanisch UK, Regen T, Eggen BJ, Boddeke HW: Histone deacetylase inhibitors suppress immune activation in primary mouse microglia. J Neurosci Res 2013, 91:1133-1142.

14. Fontan-Lozano A, Romero-Granados R, Troncoso J, Munera A, Delgado-Garcia JM, Carrion AM: Histone deacetylase inhibitors improve learning consolidation in young and in KA-inducedneurodegeneration and SAMP-8-mutant mice. Mol Cell Neurosci 2008, 39:193-201.

15. Cao Y, Ni C, Li Z, Li L, Liu Y, Wang C, Zhong Y, Cui D, Guo X: Isoflurane anesthesia results in reversible ultrastructure and occludin tight junction protein expression changes in hippocampal blood-brain barrier in aged rats. Neurosci Lett 2015, 587:51-56.

16. Barrientos RM, Hein AM, Frank MG, Watkins LR, Maier SF: Intracisternal interleukin-1 receptor antagonist prevents postoperative cognitive decline and neuroinflammatory response in aged rats. $J$ Neurosci 2012, 32:14641-14648.

17. Subhramanyam CS, Wang C, Hu Q, Dheen ST: Microglia-mediated neuroinflammation in neurodegenerative diseases. Semin Cell Dev Biol 2019.

18. Taipa R, Ferreira V, Brochado P, Robinson A, Reis I, Marques F, Mann DM, Melo-Pires M, Sousa N: Inflammatory pathology markers (activated microglia and reactive astrocytes) in early and late onset Alzheimer disease: a post mortem study. Neuropathol App/ Neurobiol 2018, 44:298-313.

19. Dantzer R, O'Connor JC, Freund GG, Johnson RW, Kelley KW: From inflammation to sickness and depression: when the immune system subjugates the brain. Nat Rev Neurosci 2008, 9:46-56.

20. Keren-Shaul H, Spinrad A, Weiner A, Matcovitch-Natan O, Dvir-Szternfeld R, Ulland TK, David E, Baruch K, Lara-Astaiso D, Toth B, et al: A Unique Microglia Type Associated with Restricting Development of Alzheimer's Disease. Cell 2017, 169:1276-1290 e1217.

21. Marty V, El Hachmane M, Amedee T: Dual modulation of synaptic transmission in the nucleus tractus solitarius by prostaglandin E2 synthesized downstream of IL-1beta. Eur J Neurosci 2008, 27:31323150.

22. Dantzer R: Cytokine-induced sickness behavior: mechanisms and implications. Ann N Y Acad Sci 2001, 933:222-234.

23. Didonna A, Opal P: The role of neurofilament aggregation in neurodegeneration: lessons from rare inherited neurological disorders. Mol Neurodegener 2019, 14:19. 
24. Kriz J, Zhu Q, Julien JP, Padjen AL: Electrophysiological properties of axons in mice lacking neurofilament subunit genes: disparity between conduction velocity and axon diameter in absence of NF-H. Brain Res 2000, 885:32-44.

25. Mattsson N, Andreasson U, Zetterberg H, Blennow K, Alzheimer's Disease Neuroimaging I: Association of Plasma Neurofilament Light With Neurodegeneration in Patients With Alzheimer Disease. JAMA Neurol 2017, 74:557-566.

26. Han Q, Lin Q, Huang P, Chen M, Hu X, Fu H, He S, Shen F, Zeng H, Deng Y: Microglia-derived IL-1 beta contributes to axon development disorders and synaptic deficit through p38-MAPK signal pathway in septic neonatal rats. J Neuroinflammation 2017, 14:52.

27. Bruns S, Stark Y, Roker S, Wieland M, Drager G, Kirschning A, Stahl F, Kasper C, Scheper T: Collagen biomaterial doped with colominic acid for cell culture applications with regard to peripheral nerve repair. J Biotechnol 2007, 131:335-345.

28. Bozkurt A, Lassner F, O'Dey D, Deumens R, Bocker A, Schwendt T, Janzen C, Suschek CV, Tolba R, Kobayashi $\mathrm{E}$, et al: The role of microstructured and interconnected pore channels in a collagen-based nerve guide on axonal regeneration in peripheral nerves. Biomaterials 2012, 33:1363-1375.

\section{Figures}


A

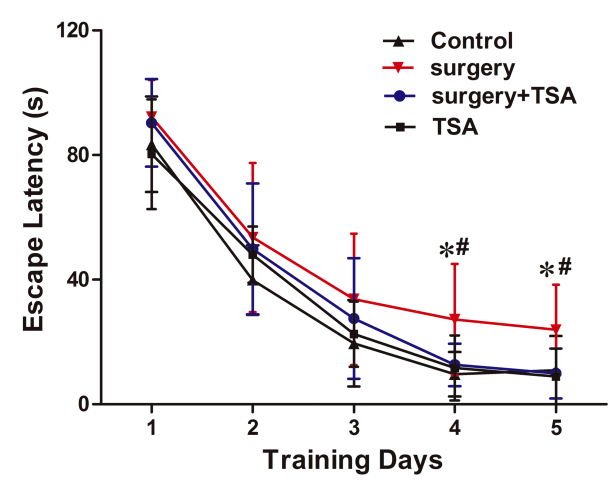

C

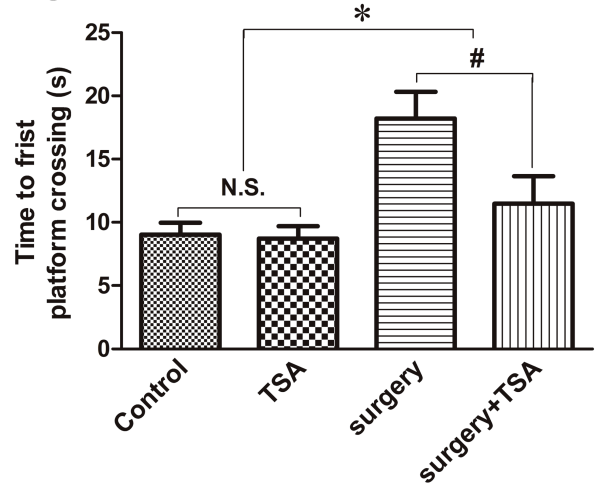

E

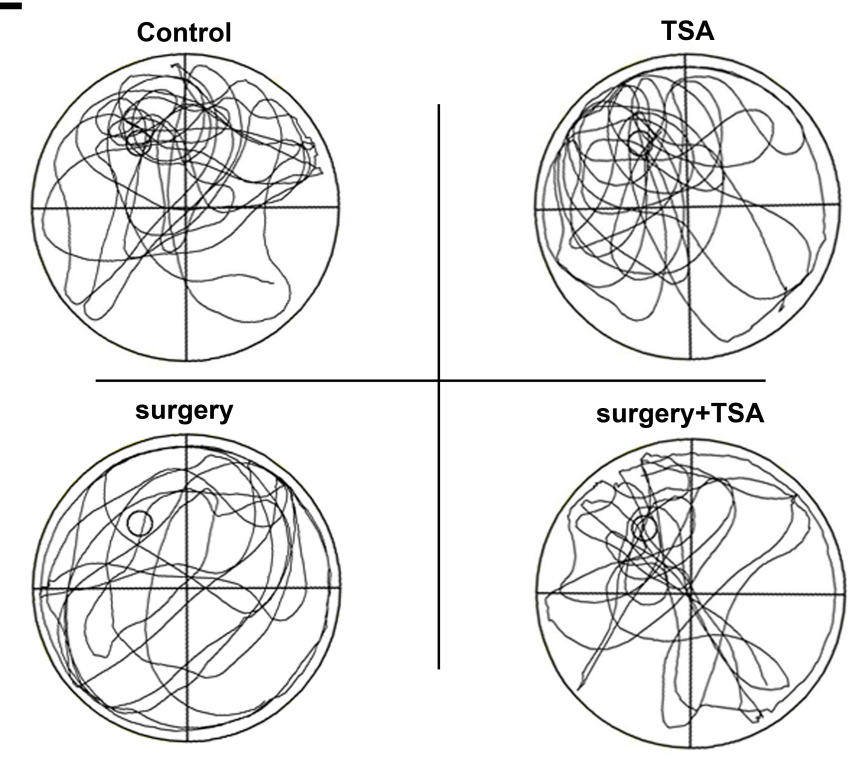

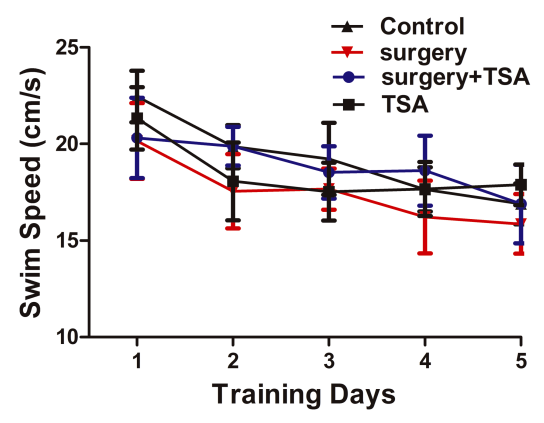

D

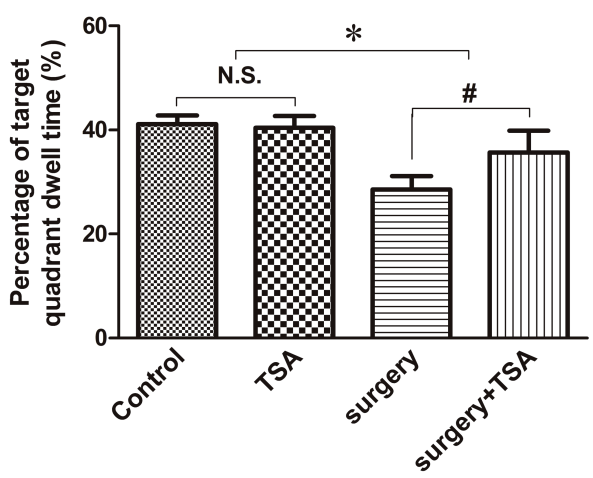

\section{Figure 1}

TSA can mitigate surgery-induced spatial learning and memory impairments in aged rats. (A, B) Acquisition trials demonstrating latencies for rats to locate the hidden platform during the 5 testing days (A) and the swimming speed (B), measuring spatial information acquisition. (C, D) On test day 6, probe trials demonstrating the time to first platform crossing (C) and time spent in the target quadrant (D), measuring memory retention capabilities. (E) Representative searching swimming paths of four aged rats 
with different treatments in the probe trial tests. Data are given as means $\pm S E M, n=12 .{ }^{*} p<0.05$, vs. the control group; \#p <0.05, vs. the surgery group.

A
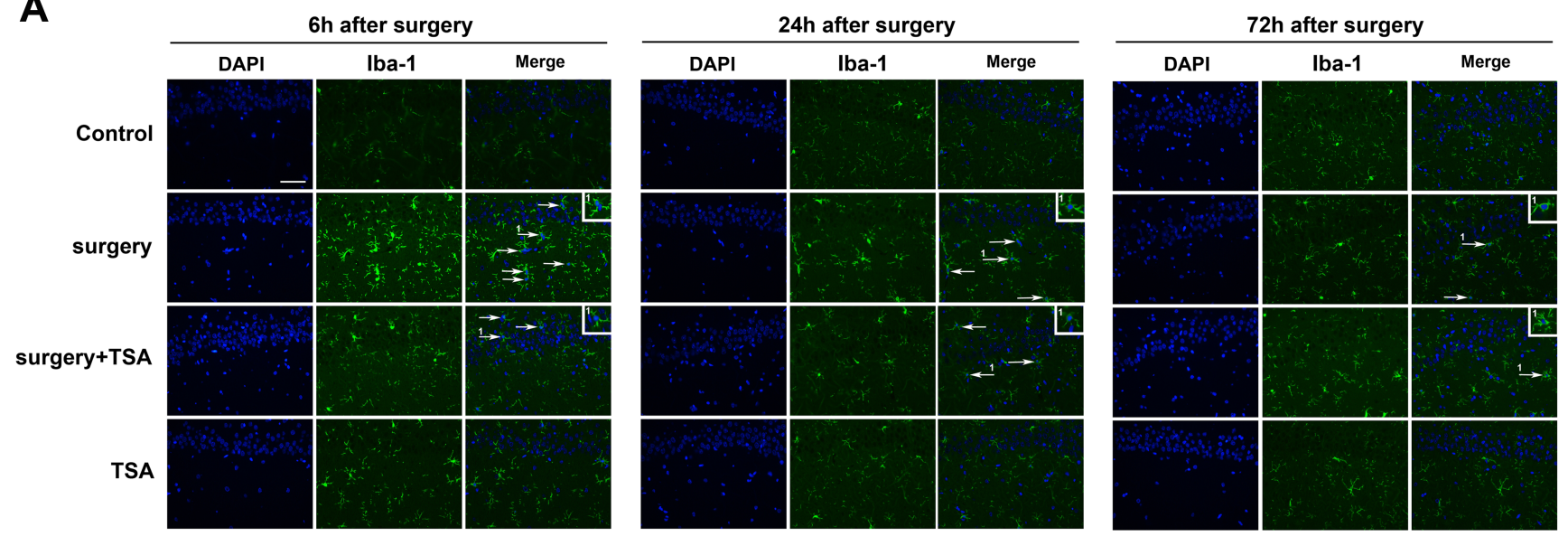

B

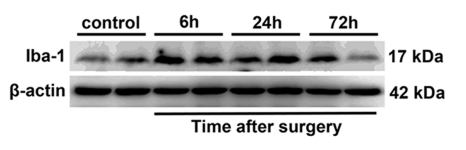

F

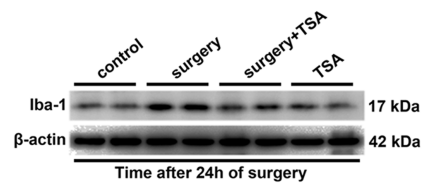

C

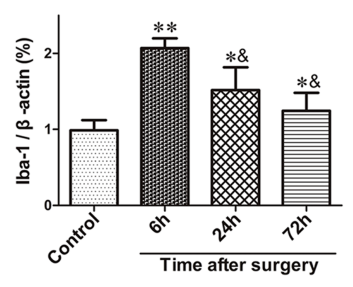

G

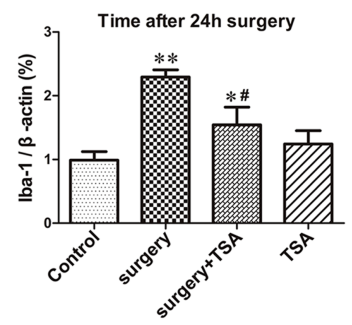

D

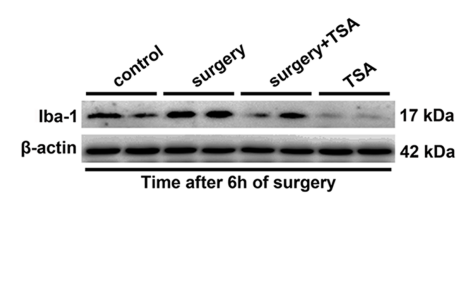

H

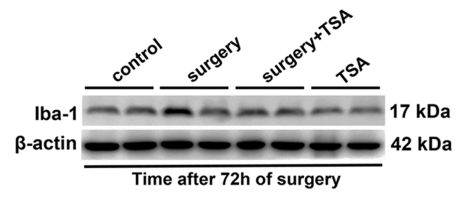

E

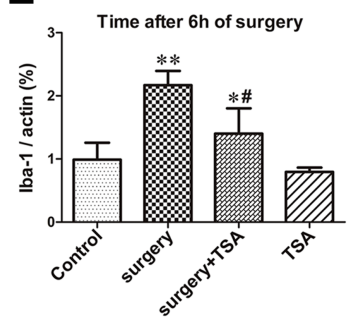

I

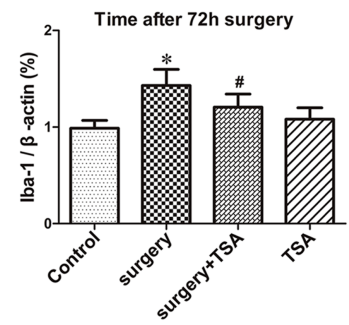

\section{Figure 2}

TSA inhibits surgery-induced hippocampal microglia activation in aged rats. (A) Immunofluorescence analysis of higher expression of Iba-1 protein in the hippocampal CA1 area were observed at postoperative $6 \mathrm{~h}, 24 \mathrm{~h}$ and $72 \mathrm{~h}$, and this staining was significantly inhibited by TSA pretreatment (Iba-1, green; cell nuclei, blue. Magnification 400x, Scale bar $=50 \mu \mathrm{m})$. Western blot analysis $(B, D, F$ and $H)$ and semi-quantitative data showing protein expression ( $C, E, G$ and I), indicating that the expression levels of Iba-1, a microglia activation marker, increased significantly at $6 \mathrm{~h}, 24 \mathrm{~h}$ and $72 \mathrm{~h}$ after laparotomy, and it peaked at $6 \mathrm{~h}$ and decreased within $72 \mathrm{~h}$ post-surgery, which was significantly inhibited by TSA pretreatment, with $\beta$-actin used as a loading control. Data are given as means $\pm S E M, n=5$. ${ }^{*}<<0.05$ and ${ }^{* * *} \mathrm{p}<0.001$, vs. the control group; $\# p<0.05$, vs. the surgery group; $\& p<0.05$, vs. 6 h after surgery. 

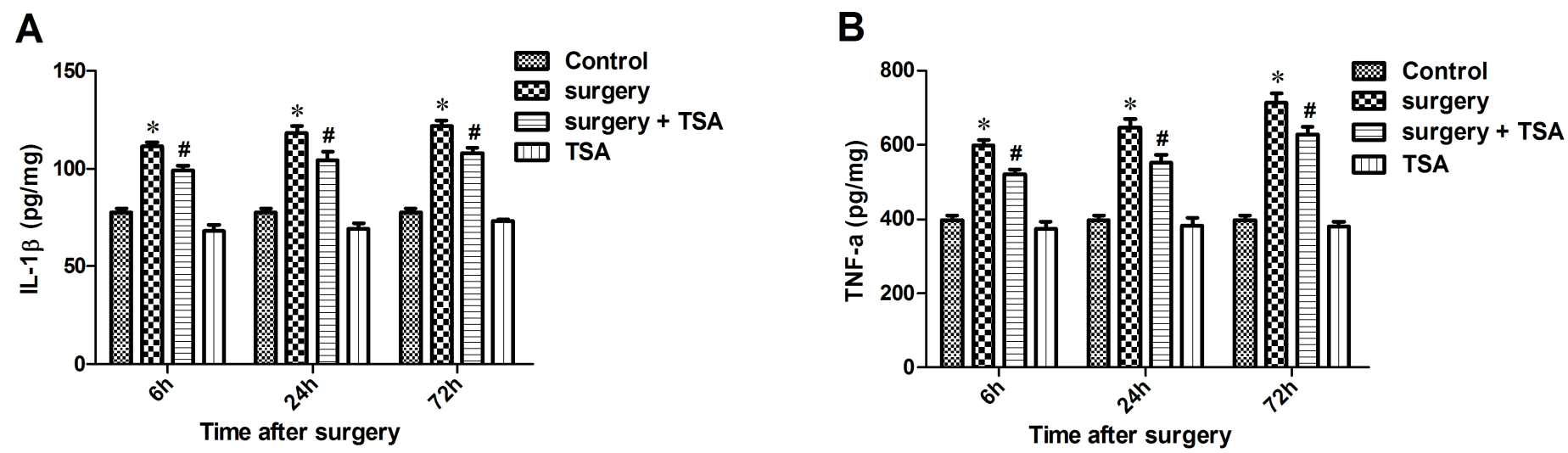

Figure 3

Effects of laparotomy on the levels of hippocampal IL-1b and TNF- $a$ in aged rats. Compared with control rats, significant changes in the levels of IL-1 $\beta$ (A) and TNF-a (B) are observed at $6 \mathrm{~h}, 24 \mathrm{~h}$ and $72 \mathrm{~h}$ after surgery, which was significantly inhibited by TSA pretreatment. Data are given as means $\pm S E M, n=5 .{ }^{*} p$ $<0.05$, vs. the control group; $\# p<0.05$, vs. the surgery group. 
Table 1 Results form iTRAQ analyses for the proteins included in targeted proteomics

\begin{tabular}{|c|c|c|c|}
\hline Proteins & Uniprot accession $\mathrm{nr}$ & Fold change $^{a}$ & $P$ value $^{\mathrm{b}}$ \\
\hline Intermed iate filament (IF) rod domain profile. (ProSiteProfiles) & IPR039008 (ProS iteProfiles) & 1.4 & 0.01570 \\
\hline Intermed iate filament protein (Pfam) & IPR039008 (Pfam) & & \\
\hline Intermediate filament (IF) rod domain signature. (ProSitePatterns) & IPR018039 (ProS itePatterns) & & \\
\hline
\end{tabular}

${ }^{a}$ Fold change in the surgery group vs. (the control, surgery + TSA and TSA groups) .

${ }^{\mathrm{b}}$ Proteins differentially expressed $(p<0.05)$ the surgery group $v s$. (the control, surgery+TSA and TSA groups)

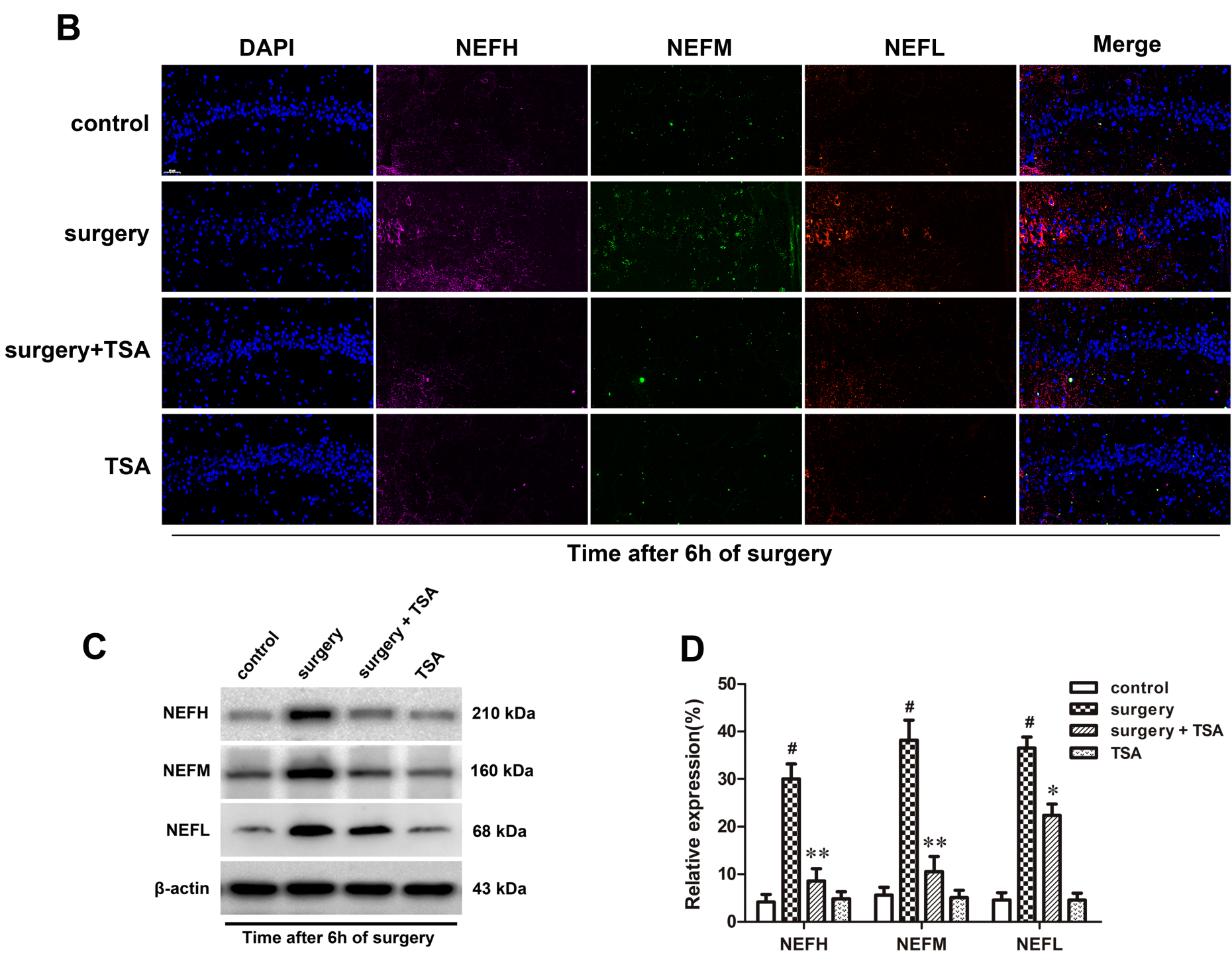

Figure 4

TSA pretreatment reduces surgery-induced upregulation of NFs in the hippocampus of aged rats. (A) iTRAQ analyses for the NFs proteins at $6 \mathrm{~h}$ post-surgery in proteomics. (B) Immunofluorescence analysis of the NFs reveals it increased in the hippocampal CA1 region at $6 \mathrm{~h}$ after surgery, which was significantly inhibited by TSA pretreatment (cell nuclei, blue; NEFH, purple; NEFM, green; NEFL, orange. Magnification $400 \times$, Scale bar $=50 \mu \mathrm{m}) .(C$ and D) Representative western blotting images and statistical analysis of 
$\mathrm{NEFH}, \mathrm{NEFM}$ and NEFL are shown. Data are given as means $\pm \mathrm{SEM}, \mathrm{n}=4 . \# \mathrm{p}<0.01$, vs. the control group; ${ }^{*} p<0.05,{ }^{*} p<0.01$ vs. the surgery group.

A

KEGG Pie Chart

(the surgery + TSA vs. the surgery group) ${ }^{6 \%}$

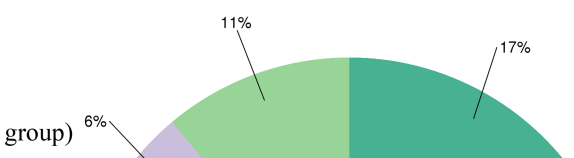

B

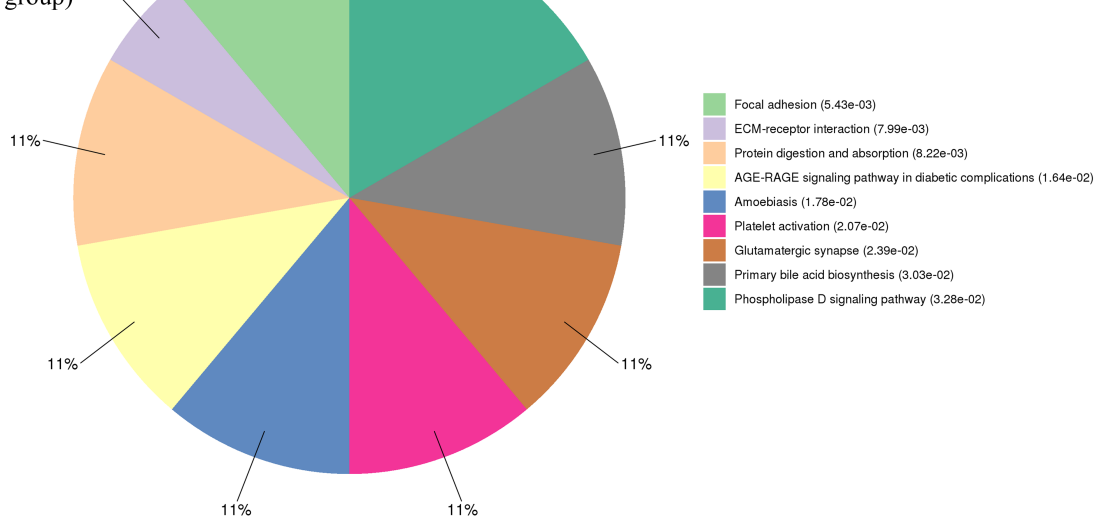

Table 2 KEGG enrichment analysis of differential expression of proteins in the surgery + TSA vs. the surgery group

\begin{tabular}{clccl}
\hline Pathway ID & \multicolumn{1}{c}{ Pathway name } & Count & $\boldsymbol{P}$ value & \\
\hline rno04510 & Focal adhesion & 3 & 0.005433 & A0A0G2K5E8, P02454, A0A0H2UHR7 \\
rno04512 & ECM-receptor interaction & 2 & 0.007986 & A0A0G2K5E8, P02454 \\
rno04974 & Protein digestion and absorption & 2 & 0.008215 & A0A0G2K5E8, P02454 \\
rno04933 & AGE-RAGE signaling pathway in diabetic complications & 2 & 0.016399 & A0A0G2K5E8, P02454 \\
rno05146 & Amoebiasis & 2 & 0.017835 & A0A0G2K5E8, P02454 \\
rno04611 & Platelet activation & 2 & 0.020688 & A0A0G2K5E8, P02454 \\
rno04724 & Glutamatergic synapse & 2 & 0.023902 & G3V6R0, P31421 \\
rno00120 & Primary bile acid biosynthesis & 1 & 0.030281 & A0A0G2K4N5 \\
rno04072 & Phospholipase D signaling pathway & 2 & 0.032756 & A0A0G2K5E8, P02454, A0A0H2UHR7 \\
\hline
\end{tabular}

C

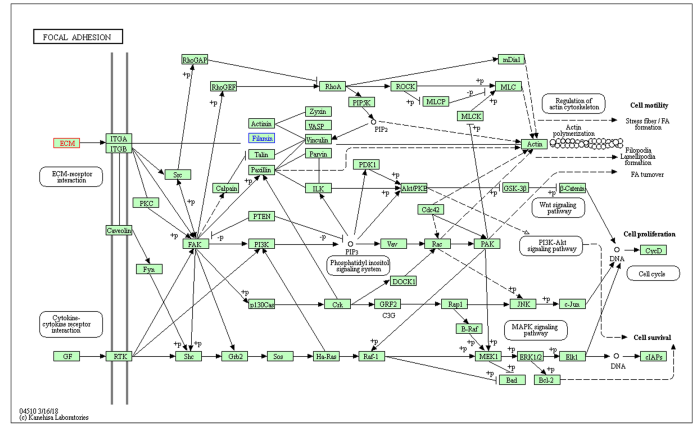

D

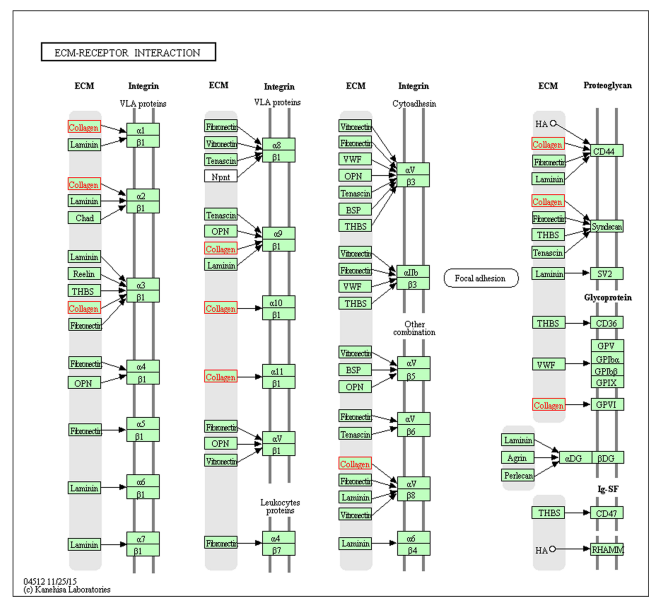

\section{Figure 5}

KEGG pathway analysis in the surgery + TSA group vs. the surgery group at $6 \mathrm{~h}$ post-surgery. (A) Top nine significantly enriched pathways identified by KEGG analysis. (B) Pathway enrichment analysis of differential expression of proteins. (C) Results show proteins involved in the focal edhesion and ECM- 
receptor interaction pathways. Red colors represent the proteins that are upregulated, and blue colors represent the proteins that are downregulated. 